\section{ДОДАТОК}

Тези

Міжнародного симпозіуму з клітинної біології та 5-го Всеукраїнського конгресу з клітинної біології

\section{Секція}

\section{Біологія стовбурових та спеціалізованих клітин і тканин}

м. Одеса, Україна

SUPPLEMENT

Conference Abstracts

International Symposium on Cell Biology jointly with $5^{\text {th }}$ Ukrainian Congress for Cell Biology

\section{Session \\ Biology of stem cells and specialized cells and tissues}

October 2-6, 2016

Odesa, Ukraine

\title{
Differentiation of pluripotent stem cells into cardyomyocytes is influenced by the seeding density of embryoid bodies
}

\author{
Budash G. V., Bil'ko N. M. \\ National University of Kyiv-Mohyla Academy, Kyiv, Ukraine \\ e-mail: Galina19@ukr.net
}

Pluripotent stem cells (PSCs), which include induced pluripotent stem cells (iPSCs) and embryonic stem cells (ESCs), have the ability to differentiate into tissues of all three germ lineages including ectoderm, mesoderm and endoderm. They can be used for disease modeling, drug toxicity screening or drug discovery, gene therapy, cell replacement therapy and other. Many human and mouse differentiation protocols begin with the formation of embryoid bodies (EBs). EBs are three-dimensional aggregates, representing the early stages of embryo development. Often formed EBs are heterogeneous in size and shape. This leads to inefficient and uncontrolled differentiation proces. In order to get homogeneous population of EBs AggreWell plates were used. Each well of the plate contains a certain number of microwells. After transferring the defined amount of single cell suspension in each of such wells EBs of a certain size are formed.

METHODS. Transgenic murine iPSC and ESC lines were differentiated into cardiomyocytes in AggreWell plates. Both cell lines were genetically modified and express IRES-flanked enhanced green fluorescent protein (eGFP) under the control of cardiac alpha myosin heavy chain promoter. We applied flow cytometry and fluorescence microscopy to test the efficiency of the differentiation processes.

RESULTS. At the 1-st day of differentiation we formed EBs with the seeding density of 250, 500, 750, 1000 and 2000 iPSCs in each EB. It was found that the efficiency of differentiation of EBs with 250 and 1000 cells is much lower compared with EBs formed of 500 and 750 cells. EBs the size of which was 2000 cells per each body produced no cardiomyocytes. On the 13-th day of differentiation when the highest amount of cardiomyocytes is obtained, the number of GFP+ cells derived from EBs with seeding density of 500 cells was 8.5 times higher than from EBs with 250 cells; and 4.7 times higher than from EBs formed starting with 1000 cells. The difference between 500 and 750 cells EBs was $30 \%$. When AggreWell plates were used for ESCs lines differentiation no GFP+ cells or beating areas were observed. The EBs of different size and shape were identified on the second day of differentiation. Adding of cardiogenic enhancers such as ascorbic acid, DMSO, G-CSF, dorsomorphin did not provide positive effect. Applying DMSO and dorsomorphin even had opposite effect - the amount of GFP+ ${ }^{+}$cells decreased 
comparing with differentiation without supplements. Differentiation of 500 iPSCs EBs provided in AggreWell plates (where the seeding density and homogeneity of EBs size is under scientist's control) was $80 \%$ higher than differentiation in rotating suspension culture (where EBs of different shape and size are formed spontaneously).

CONCLUSIONS. Modification of such parameters as seeding density and embryonic bodies' size can beneficially effect differentiation of induced pluripotent stem cells into cardiomyocytes. Among the embryonic bodies formed within range from 250 cells to 2000 cells per embryonic bodies the highest percentage of $\mathrm{GFP}^{+}$cells was obtained from embryonic bodies formed with 500 cells.

\title{
Subacute hematological toxicity of protein kinases inhibitor maleimide derivative with antitumor activity
}

Byelinska I., Tsyvinska S., Rybalchenko V.

Taras Shevchenko National University of Kyiv, Kyiv, Ukraine

e-mail: byelinska@univ.kiev.ua

Maleimide derivative (MI-1, 1-(4-Cl-benzyl)-3-Cl-4- $\left(\mathrm{CF}_{3}\right.$-phenylamino)-1H-pyrrole-2,5-dione synthesized in Taras Shevchenko National University of Kyiv, Ukraine) is a competitive inhibitor of VEGFR-1,2,3, FGF(h), PDK1, Src(h), Syk(h) and other protein kinases. MI 1 in vitro inhibits the proliferation of the colon cancer cells (HCT-116, SW-620) and in vivo decreases the number of colon tumors and normalizes an increased number of monocytes and platelets in blood of rat with 1,2-dimethylhydrazine-induced colon carcinogenesis. MI-1 inhibits proliferation of neoplastic monoblast cells U-937 through induction of apoptosis and inhibition of mitotic activity of cells, causes shift of cells from $\mathrm{G}_{2} / \mathrm{M}_{+} \mathrm{S}$ to $G_{0} / G_{1}$ stage. The aim of this study was to examine the effects of increased doses of Ml-1 on the blood cells of healthy rats.

Methods. The white outbred male rats with initial weight 130-140 g, were randomly divided into four groups (5-9 rats per group) and 14 days per os daily treated with: I (control) $-0.1 \mathrm{ml}$ sunflower oil containing $15 \%$ of DMSO; II $-2.7 \mathrm{mg} / \mathrm{kg}$ of Ml- 1 (effective dose, decreases the number and size of colon tumors in 1,2 dimethylhydrazine-induced colon carcinogenesis in rats, $10^{-4} \mathrm{~mol} / \mathrm{L}$ in the blood); III $-13.5 \mathrm{mg} / \mathrm{kg}$ of MI-1 (5-fold dose, $\sim 5 \cdot 10^{-4} \mathrm{~mol} / \mathrm{L}$ ); IV $-27 \mathrm{mg} / \mathrm{kg}$ of MI-1 (10-fold dose, $\sim 10^{-3} \mathrm{~mol} / \mathrm{L}$ ) each dissolved in $0.1 \mathrm{ml}$ of the sunflower oil containing 15 $\%$ DMSO. Rats were euthanized by $\mathrm{CO}_{2}$ inhalation and sacrificed by cervical dislocation. Blood cells parameters were determined by conventional methods.

The difference between the parameters was evaluated by Kruskal-Wallis test for multiple comparisons followed by nonparametric MannWhitney test using SPSS 16.0 for Windows. The median $\left(25^{\text {th }} ; 75^{\text {th }}\right.$ percentiles $)$ are presented. The difference was considered at $p<0.017$.

RESULTS. Administration of Ml-1 in a dose of $2.7 \mathrm{mg} / \mathrm{kg}$ for 14 days doesn't change blood cells parameters. Increases the dose of $\mathrm{Ml}-1$ to $13.5 \mathrm{mg} / \mathrm{kg}$ trend to decreases in leukocytes $(13.60(12.05 ; 16.06) ; p=0.059)$ and monocytes number $(0.87(0.58 ; 1.04) ; p=0.027)$ and to $27.0 \mathrm{mg} / \mathrm{kg}-(14.35(11.78 ; 15.45) ; p=0.038)$ and $(0.72(0.49 ; 0.93) ; p=0.021)$ vs. control group $(16.05(15.25 ; 18.00)$ and $1.21(0.91 ; 1.56))$ respectively.

$\mathrm{Ml}-1$ at the doses $13.5 \mathrm{mg} / \mathrm{kg}(654.59(599.90 ; 703.31)$ and $27.0 \mathrm{mg} / \mathrm{kg}(652.29(544.83 ; 780.52)$ decrease in platelets number $(p=0.012 ;$ $\mathrm{p}=0.027$ respectively) vs. control group (808.00 (768.11; 925.38$)$.

CONCLUSIONS. MI-1 at the doses of 13.5 and $27 \mathrm{mg} / \mathrm{kg}$ after 14 day of administration causes trend to decreases in leukocytes, monocytes and platelets number that may indicate alteration of hematopoiesis.

\section{AKAP9 and DOCK7 are binding partners of myosin VI}

\author{
Chumak V. ${ }^{1,2}$, Sobczak M. ${ }^{1}$, Pomorski P. ${ }^{1}$, Suszek M. ${ }^{1}$, Redowicz M. J. ${ }^{1}$ \\ ${ }^{1}$ Nencki Institute of Experimental Biology, Warsaw, Poland \\ ${ }^{2}$ Institute of Cell Biology NAS of Ukraine, Lviv, Ukraine \\ e-mail: virachumak@gmail.com
}

Myosin $\mathrm{VI}(\mathrm{MVI})$ is a unique motor protein that moves unlike other myosins towards the minus end of actin filaments. MVI is involved in cell migration and adhesion, endocytosis, intracellular trafficking, cargo sorting and cell migration. We showed that AKAP9 [Karolczak et al., 2015] and DOCK7 [Majewski et al., 2012] are binding partners of MVI. AKAP9 belongs to the family of A-Kinase Anchor Proteins (AKAPs) which have the common function of binding to the regulatory subunit of protein kinase A (PKA). Altered AKAP activity is implicated in many pathological processes including cardiovascular disorders, immune diseases, and multiple cancer phenotypes. Another MVI binding partner DOCK7 functions as a guanine nucleotide exchange factor (GEF), which activates Rac1 and Rac3 Rho small GTPases by exchanging bound GDP for free GTP. DOCK7 is involved in the regulation of cortical neurogenesis through the control of radial glial cells proliferation versus differentiation.

Two regions of MVI cargo domain were found to be involved in a partner recognition: a positively charged RRL motif (aa 1115-1117) and a hydrophobic WWY (aa 1191-1193) motif. We tested the nature of interaction of MVI with its interaction partners with the use of MVI tail (aa 840-1284) with mutations in either of the two regions (RRL to AAA and WWY to WLY) that were cloned into pEGFP-C3 plasmids*. 
We showed in vitro that AKAP9 bound to the MVI WWY region, thus via hydrophobic interaction. We plan to test which of the AKAP9 domains is engaged in the interaction and what is the physiological relevance of this interaction, especially that an increase in AKAP9 level in muscle from Snell's waltzer mice lacking MVI ${ }^{* \star}$ was observed.

DOCK7 binds to the MVI RRL region, thus via electrostatic interactions. Moreover, we showed that MVI bound to D0CK7 C-terminal domains of M2 and DHR2, involved in the protein-protein interactions and GEF activity, respectively. We also demonstrated that in PC12 cells this interaction was important for NGF-stimulated outgrowth formation.

Notes: *tail mutants and ${ }^{* *}$ Snell's waltzer mice are gifts from Dr. Folma Buss from MRC in Cambridge

\title{
Proliferative potential of cancer stem cells at various stages of breast cancer: experimental study
}

\author{
Goltsev A. M., Bondarovych M. O., Ostankov M. V., Chelombitko O. V., Ostankova L. V. \\ Institute for Problems of Cryobiology and Cryomedicine NAS of Ukraine, Kharkiv, Ukraine \\ e-mail: cryopato@gmail.com
}

It is known that manifestation of breast cancer is determined by the degree of proliferative activity of cancer stem cells (CSCs). Thereby it should be important to estimation of cell's proliferation in the normal mammary gland and breast cancer for diagnosis and treatment of the cancer patients.

AIM. To evaluate the proliferative potential of the CSCs of various differentiation degree in mammary gland, in the tumors of various sizes and in the lung metastases.

MATERIALS AND METHODS. Our experiments were carried out on the 16-month old C3H and CBA male mice weighing 18-20 g. The mice were divided into the following groups: 1 - CBA (control); $2-\mathrm{C} 3 \mathrm{H}$ without tumor; $3-\mathrm{C} 3 \mathrm{H}$ with tumor of $0.5-1.0 \mathrm{~cm}$ in diameter; $4-\mathrm{C} 3 \mathrm{H}$ with tumor of $1.5-2.0 \mathrm{~cm} ; 5$ - with tumor and lung metastases. The number of CSCs in tissue was estimated by FACS using monoclonal antibodies to CD24, CD44 and CD133.

RESULTS. In group 2 in the mammary gland tissue there was an increased number of the most invasive (CD44 hi) and more differentiated $\left(\mathrm{CD} 44^{+} / 24^{-}\right.$and $\left.\mathrm{CD}^{3} 3^{+}\right)$of cancer cells in the comparison with control group. In the group 3 the number of CD44 and CD44 $/ 24^{-}$cells in the tumor was increased whereas the number of $\mathrm{CD} 133^{+}$was decreased in the comparison with group 2. However the number of CSCs with these markers in the tumor of group 3 was lower than in the mammary gland.

In the group 4 the number of $\mathrm{CD} 44^{\mathrm{hi}}, \mathrm{CD} 44^{+} / 24^{-}$and $\mathrm{CD} 133^{+}$cells in the tumor was greater than in the group 3 . But in mammary gland the $\mathrm{CD} 44^{\mathrm{hi}}$ and $\mathrm{CD} 44^{+} / 24^{-}$cells decreased and the number of $\mathrm{CD} 133^{+}$cells remained the level of group 3.

In the group 5 versus group 4 the number of $\mathrm{CD} 44^{\mathrm{hi}}$ and $\mathrm{CD} 133^{+}$cells in the tumor and mammary gland was decreased The number of $C D 44^{+} / 24^{-}$cells in the tumor in this group was decreased and it was increased in the mammary gland tissue that can evidence about redistribution of the CSCs proliferative potential. The number of $\mathrm{CD}_{133^{+}}$cells in lung metastases was decreased and the content of CD44 and $\mathrm{CD} 44^{+} / 24^{-}$cells was increased relative to the tumors in this group that can display various tropicity of CSCs during metastasing into the lung.

CONCLUSIONS. Our results revealed redistribution of the CSCS of various differentiation degree during tumor progression. We can recommend our method of CSCs estimation in the mammary gland as a diagnostic test in the choice of treatment tactic for cancer patients.

\section{Evaluation of L-canavanine influence on morphology and migration of human glioblastoma cells under arginine deprivation}

\author{
Karatsai O. ${ }^{1}$, Stasyk O. ${ }^{2}$, Rędowicz M. J. ${ }^{1}$ \\ ${ }^{1}$ Nencki Institute of Experimental Biology PAS, Warsaw, Poland \\ ${ }^{2}$ Institute of Cell Biology NAS of Ukraine, Lviv, Ukraine \\ e-mail: o.karatsai@nencki.gov.pl
}

The strategy of enzymatic degradation of certain amino acids is one of the promising approaches of modern anticancer therapy. This therapy is considered as nontoxic and selective since it allows controlling growth of malignant tumors deficient in arginine biosynthesis. We propose that arginine deprivation-based combinational treatment with the plant origin arginine analogue L-canavanine can enhance anticancer effect of arginine depletion. Malignant brain tumors are hardly amenable to conventional chemotherapies. Glioblastomas can be potential targets of arginine deprivation-based enzymotherapy as it is not dependent on permeability of the brain-blood barrier.

We compared the cytotoxic effect of L-canavanine for human U251MG and U87MG glioblastoma cells as experimental models. We observed that under arginine deprivation canavanine strongly inhibited the viability of cells in the concentration- and time-dependent manner. The cytotoxic concentration of canavanine is profoundly lower under the arginine deficiency because $\mathrm{L}$-canavanine can incorporate into proteins instead of $\mathrm{L}$-arginine during ribosomal biosynthesis thus causing alterations in the protein structure. This may lead to inhibition of cell growth and cause cell death. 
We noted that after $48 \mathrm{~h}$ of cell cultivation in the absence of arginine but in the presence of canavanine significant changes in morphology of the examined U251MG and U87MG cells were visible, especially in the organization of the leading edge actin-rich structures such as lamellipodium and fillopodia. These alterations may be responsible for the observed halt of cell penetration through the porous membrane using the Transwell system. It should be emphasized that we did not observe any changes control isolated rat glia cells subjected to the same experimental regime as the glioblastoma cells.

Thus our data seem to indicate that a combination of arginine deprivation with canavanine treatment may be indeed considered for the development as a new therapeutic approach against highly malignant glioblastomas.

\title{
Mesenchymal stem cells express nicotinic acetylcholine receptors
}

\author{
$\underline{\text { Koval L. }}{ }^{1}$, Deryabina O. ${ }^{2}$, Ruban T. ${ }^{3}$, Ustymenko A. ${ }^{2}$, Kyryk V. ${ }^{2}$, Skok M. ${ }^{1}$ \\ ${ }^{1}$ Palladin Institute of Biochemistry NAS of Ukraine, Kyiv, Ukraine; \\ ${ }^{2}$ State Institute of Genetic and Regenerative Medicine NAMS of Ukraine, Kyiv, Ukraine; \\ ${ }^{3}$ Institute of Molecular Biology and Genetics NAS of Ukraine, Kyiv, Ukraine
}

Nicotinic acetylcholine receptors (nAChRs) are ligand-gated ion channels mediating fast synaptic transmission in muscles and autonomic ganglia, regulating neurotransmitter release in the brain and controlling various functions in non-excitable cells. Actually, cholinergic regulation is one of the ancient mechanisms regulating survival, proliferation and motility of eukaryotic cells.

Mesenchymal stem cells (MSCs) are multipotent early progenitors of different cell types like chondrocytes, adipocytes, fibroblasts and neurons. Currently, they are explored and regarded as promising therapeutic agents for various pathological states including inflammatory and degenerative disorders. The aim of our study was to reveal if MSC express nAChRs and, therefore, can be subjected to regulation by acetylcholine.

We explored MSCs purified from either human umbilical cord (huMSCs) or mouse placenta (mpMSCs) using both monoclonal and polyclonal antibodies specific to various nAChR subunits by flow cytometry, ELISA and immunocytochemistry followed by confocal microscopy. It was found that both huMSCs and mpMSCs express a3-, a7- and a9-containing nAChRs, and their density on the cell is being changed depending on the cell state. In particular, the general increase of nAChR density and re-distribution of nAChR subtypes in favor of a7-containing ones has been observed along with the MSCs cultivation in vitro. It was found that MSCs, which had been frozen and thawed express less nAChRs than non-frozen cells. Finally, huMSCs pushed to differentiation into chondrocytes down-regulated their nAChR expression.

The data obtained indicate that the nAChRs are found at the very early stages of cell differentiation and, therefore, can mediate the regulating effects of cholinergic ligands like choline and acetylcholine, as well as the influence of exogenous nicotine. The level of these receptors can characterize the MSCs state that should be taken into account upon MSCs transplantation.

\section{Mesenchymal stem cells development and function}

\author{
Lisyany M. I. \\ The State Institution "Romodanov Neurosurgery Institute, National Academy of Medical Sciences of Ukraine", Kyiv, Ukraine
}

The mesenchymal stem cells of adult organisms are capable not only to differentiate into specialized cells but they can also regulate the proliferation of other cells, including immune system. The various tissues are a source of stromal cells capable for adhesion to the plastic and proliferation into the fibroblast-like cells. There is no universal protocol of MSCs harvesting and time in culture and the number of passages are different (from 2-3 to 10-12 passages) indicating that passing of definite stages of MSCs transformation. We identified minimum four such stages of MSCs development and respectively four types of various MSCs progenitors: primary adhesive fibroblast-like MSCs (dormant nonproliferating), activated proliferating fibroblasts ( $2^{\text {nd }}$ passage), MSCs progenitors (rapidly proliferating) and mature MSCs, which are capable for differentiation in the certain direction. So far none of the stages of MSCs development has been distinctly characterized and, probably, each type of the MSCs progenitors has its own phenotypic and functional properties which can be realized via contact interaction and synthesis of humoral factors.

Our investigations showed that supernatants of 24-48-hour adhesive adipose-derived cell culture (first passage) inhibit phytohemagglutinininduced lymphocytes proliferation, whereas the supernatants of second and third passage stimulate spontaneous and induced proliferation of the lymphocytes.

The functional difference between various types of MSCs progenitors was also demonstrated on the model of skin wound healing in the rats. The local injection of first passage (48 hours in culture) adhesive cells inhibited regeneration and healing of the wounds whereas cells of 3-4 passage (15-25-day culture) stimulated wound healing.

CONCLUSIONS. Our results agree the numerous literature data demonstrating different biological effects that are induced by the bone marrow and adipose-derived stromal cells, depending on the time of culture. Further investigation of MSCs development stages and establishing their functional characteristics will allow obtain the cells and their humoral factors with the required properties that are very essential both, in experiment and clinical study. 


\title{
The effect of the fetal neural cells supernatant in cultured C6 glioma cells
}

\author{
Liubich L. D., Lisyany N. I., Semenova V. M., Malysheva T. A., Stayno L. P., Vaslovych V. V. \\ The State Institution "Romodanov Neurosurgery Institute, National Academy of Medical Sciences of Ukraine", Kyiv, Ukraine \\ e-mail: lyubichld@gmail.com
}

Glial tumors are the largest group among the primary tumors of the central nervous system that are difficult to treat because of invasiveness and high incidence of the prolonged growth. In neurooncology the criteria of individualization of patients treatment are actively developed on the basis of identifying a unique set of molecular changes in tumor cells - personalized or target therapy. As targets for brain tumors the markers of proliferation as well as neuroepithelial stem cell markers, regulators of cell proliferation, differentiation and survival/apoptosis are considered. Cellular and molecular similarities between brain tumor stem cells (BTSCs) and normal neurogenic stem cells (NSCs) substantiates the search for new methods of treatment of malignant glioma using NSCs [Aboody K. S. et al., 2013; Bovenberg M. S. et al., 2013; Shah Kh. et al., 2013; Morshed R. A. et al., 2015]. We previously reported the antiproliferative effect of rat neurogenic fetal cells supernatant (RNFCS): the number of Ki-67-immunopositive tumor cells under the influence of RNFCS high statistically decreased (2.7 fold) [Liubich L. D. et al., 2015]. Since CD133 molecule could be one of the most characteristic markers of BTSCs and considered as a target for therapy of brain tumors [Angelastro J. M. et al., 2010], the aim of the present paper was to study the action of RNFCS on the content of CD133-immunopositive cells in C6 glioma cell cultures.

METHODS. The cells of rat brain glioma (cell line $\mathrm{C6}, \mathrm{n}=16$ ) served as the material for culturing. RNFCS was obtained from suspensions of neurogenic rat brain cells on 14th (E14) day of gestation. The study was performed in control cultures (standard cultivation conditions without RNFCS adding) and research cultures (with adding RNFCS $(0.10 \mathrm{mg} / \mathrm{ml}$ ) for 48 hours). Immunocytochemical staining for CD133 molecule (one of the markers of neural epithelial stem cells) was performed using murine monoclonal antibodies to CD133 (Millipore, USA), immunocytochemical staining for the proliferation marker Ki-67 - using rabbit monoclonal antibodies to Ki-67 (Thermo Scientific, USA). In cytological specimens the changes in cellular composition and the number of immunopositive cells were determined.

RESULTS. Under the conditions of RNFCS action at a concentration of $0.10 \mathrm{mg} / \mathrm{ml}$ for 48 hours the number of CD133-immunopositive cells in C6 rat glioma cell cultures was reduced ( 4.2 fold; $p=0.02$ compared to control, U-Mann-Whitney test). CD133-immunopositive cells in C6 glioma culture had a smaller size than immunonegative cells (average values of cell sectional area, nucleus cell-sectional area) and greater nuclearcytoplasmic ratio.

Spearman rank correlation analysis doesn't show the statistically positive correlation between amount of CD133-immunopositive BTSCs and Ki-67-immunopositive cells in C6 glioma under the influence of RNFCS $(r=0.49, p=0.17)$, but reveals the significant inverse correlation between CD133-immunonegative and Ki-67-immunopositive cells ( $r=-0.71, p=0.03$ ).

CONCLUSION. Changes in expression of neural epithelial stem cells marker by cells of C6 glioma monoculture under the influence of RNFCS may become a precondition for theoretical foundation of new approaches to complex pathogenetic therapy in brain glioma patients using preparations derived from fetal neurogenic cells.

\section{The role of carbohydrate component of recombinant $a 7$ nicotinic acetylcholine receptor extracellular domain in its immunogenicity and functional effects of resulting antibodies}

\author{
Lykhmus O. ${ }^{1}$, Koval L. ${ }^{1}$, PastuhovaD. ${ }^{1}$, Zouridakis M. ${ }^{2}$, Tzartos S. ${ }^{2}$, Komisarenko S. ${ }^{1}$, Skok M. ${ }^{1}$ \\ ${ }^{1}$ Palladin Institute of Biochemistry NAS of Ukraine, Kyiv, Ukraine; \\ ${ }^{2}$ Hellenic Pasteur Institute, Athens, Greece \\ e-mail: elenalykhmus@yandex.ua
}

Nicotinic acetylcholine receptors of a7 subtype ( $\mathrm{a} 7 \mathrm{nAChRs}$ ) attenuate the inflammatory cytokines production by macrophages and are involved in pathogenesis of Alzheimer disease by directly influencing the processing of amyloid-beta (A $\beta$ ) precursor protein in the brain. Previously we found that regular injections of bacterial lipopolysaccharide (LPS) decreased the level of a7 nAChRs and stimulated accumulation of $A \beta$ peptide (1-42) in the brain of mice resulting in memory impairment. Similar effects were observed in mice immunized with recombinant extracellular domain (1-208) of a7 nAChR subunit. However, the mechanism of inflammation-like effect of a7-specific antibodies remained unclear.

The aim of the present study was to reveal the impact of carbohydrate component of recombinant $a 7(1-208)$ produced in yeast in the functional effect of resulting antibodies. For this purpose, C57BI/6 mice were immunized with either initial a7(1-208) or with that pre-treated with endoglycosidase. Control groups of mice obtained injections of either LPS or complete Freund's adjuvant. Mice were tested for memory performance, their blood sera were examined for the presence and fine specificity of a7(1-208)-specific antibodies and the brain preparations were studied for the levels of $a 7 \mathrm{nAChR}, A \beta(1-42)$ and interleukin-6. It was found that the original $a 7(1-208)$ was more immunogenic than the deglycosylated one, and their epitopes were recognized with different efficiency. In contrast to LPS and original a $7(1-208)$, deglycosylated a7(1-208) did not stimulate interleukin-6 elevation in the brain, i.e. had no pro-inflammatory effect. Nevertheless, immunizations with either the 
original or deglycosylated $\alpha 7(1-208)$ resulted in similar decrease of a7 nAChRs, accumulation of $A \beta(1-42)$ in the brain and significant episodic memory decline, comparable to those exerted by LPS injections.

We conclude that the decrease of a7 nAChR density, caused by a7(1-208)-specific antibody, is critical for A (1-42) accumulation and episodic memory impairment, while pro-inflammatory capacity of a7(1-208)-specific antibody plays a secondary role for the development of Alzheimer-like symptoms.

\title{
A role for unconventional myosin VI in the nervous system: new facts and hypotheses
}

\author{
Rędowicz M. J. \\ Nencki Institute of Experimental Biology, Warsaw, Poland \\ e-mail: j.redowicz@nencki.gov.pl
}

Several unconventional myosins are expressed in the nervous system where they are postulated to play important roles in the neuronal transmission and neurogenesis. One of them is myosin $\mathrm{VI}(\mathrm{MVI})$ that is a unique as it moves backwards on actin filaments. This molecular motor fulfills its functions via interaction of its N-terminal motor domain with actin along with interaction of its C-terminal globular tail domain (also termed as a cargo domain) with cell/tissue-specific partners. It acts as a cargo transporter along actin filaments and/or as an anchor linking cargo to the actin cytoskeleton. MVI was shown to be involved in numerous cellular processes including intracellular vesicular transport and transcription. With the use of Snell's waltzer (SV) mouse strain not able to express MVI (thus serving as natural MVI-KO mouse) several defects in the brain function were observed. There was a profound gliosis, impairment of synapse formation as well as inhibition of receptor internalization and neuronal transmission, and BDNF-stimulated neurotransmitter release. These changes indicate substantial defects in processes related to endocytosis and secretion.

Our recent finding that one of MVI binding partner in neurosecretory PC12 cells is DOCK7 points to yet another mechanism, namely involvement of MVI in the actin cytoskeleton organization [Majewski $Ł$. et al., 2012]. DOCK7 is a guanine exchange factor (GEF) acting on Rac small GTP binding proteins that are main regulators of the cytoskeleton dynamics. Moreover, DOCK7 was shown by others as important factor in neuritogenesis. We further characterized this interaction in PC12 cells and showed that C-terminal domains of DOCK7 bind to MVI cargo domain via electrostatic interactions and the presence of full length MVI is necessary for NGF-stimulated protrusion formation [Sobczak M. et al., 2016]. Moreover, depletion of MVI affected activity of DOCK7 and impaired its peripheral localization. We also show that MVI and DOCK7 interact in cultured rat neurons and this interaction, maintained during the 3-week culture, also affects GEF activity and neurite formation.

Moreover, we observed that DOCK7 localization is changed in SV brains though its level does not vary from the controls. These data indicate that MVI-DOCK7 interaction has functional implications in neuritogenesis, possibly by affecting organization of the actin cytoskeleton that is indispensible for the neurite initiation and elongation.

\section{HSP90 and ERK1/2 form a complex in mouse cardyomyocytes}

\author{
Roiuk M. V., Kroupskaya I. V., Pavlyuk O. V., Yakovenko L. F., Osmanova K., Sidorik L. L. \\ Institute of Molecular Biology and Genetics NAS of Ukraine, Kyiv, Ukraine \\ e-mail: sidorik@imbg.org.ua
}

Loss of function and death of cardiomyocytes is one of the major contributing factors in the development of heart failure, in which apoptosis make the significant contribution into cardiomyocytes degradation. Molecular chaperons could be molecular switches between pro- and antiapoptotic signals by its modulation of kinase activity. One of the classes of molecular chaperons is HSP90, with high substrate specificity, which can be synthesized constantly and in response to stress. One client-group proteins which need HSP90 for maturation, is protein kinases. The extracellular-regulated kinase 1/2 (ERK1/2) signalling is a key pathway in cardiomyocyte hypertrophy and survival in response to many different stress stimuli, potentiates the catalytic activity of some proapoptotic proteins such as DAP kinase in the cytoplasm.

Based on fact of HSP90 could mediate the oxidative stress-stimulated, late-phase activation of ERK1/2, proliferation by promoting the ERK $1 / 2$ phosphorylation, the association of itself with phosphor-ERK1/2, it's solubility and nuclear translocation, we suggested possibility of interaction between HSP 90 and ERK1/2 kinase in heart tissue.

Obtained from docking results, we identified the potentially interacting with HSP90 amino acids localized on the chaperon' $\mathrm{N}$ and $\mathrm{M}$ domains. For further confirmation of bioinformatically obtained results the experimental part of investigation has been done.

HSP90 domains cloned in vector pGEX4T3 were expressed in E. coli strain BL21(DE3). Verification of interaction between HSP90 domains and ERK1/2 in total lysates obtained from mice normal heart tissue was conducted by using GST pull-down assay. Precipitated ERK1/2 kinase was detected via Western blot analysis using specific anti-ERK1/2 antibodies.

Using of GST pull-down assay predicted involvement of $\mathrm{N}$ and M domains of HSP9O in the interaction with ERK1/2 kinase was confirmed. Such details like co-locolization of complex and its value for development of cardiomyocyte's apoptosis need further investigation. 


\title{
Biology of adipose tissue mesenchymal stem cells and their capacity to neuroglial differentiation
}

\author{
Semenova V. M., Lisyany M. I., Staino L. P., Belska L. M., Egorova D. M. \\ The State Institution "Romodanov Neurosurgery Institute, National Academy of Medical Sciences of Ukraine", Kyiv, Ukraine \\ e-mail: seveme22@rambler.ru
}

A priority goal of modern neurobiology and medicine is the development of cell-based therapy for treatment of serious central nervous system diseases. With this object in view, studies were carried out with the use of mesenchymal stem cells (MSCs) obtained from the adipose tissue, and their following neurogenic differentiation in vitro. Their following allo- or autotransplantation to the patients is thought to be a promising cell replacement therapy for intervertebral disc degeneration, multiple sclerosis, spinal cord injury, peripheral nerves degeneration, Huntington's disease, Parkinson's disease, Alzheimer's disease, etc. Regenerative functions of the MSCs are determined by their potency, ability to migration into the damaged tissue, adhesive properties, inhibition of immune reactivity, as well as their regulatory function and capacity for directed differentiation.

The protocols of isolation, cultivation and differentiation of adipose-derived MSCs in the osteo-, adipo-, myo-, chondroogenic and neurogenic direction were developed. Neurogenic differentiation of adipose-derived MSCs appeared to be accompanied with an increased expression of the specific genes and cytoskeletal markers: nestin (marker of early neural progenitors), neuron-specific tubulin-3, glial fibrillar acidic protein (GFAP), microtubule-associated protein MAP-2, neuron specific enolase Eno2 and others that confirm their neuronal phenotype. The signs of neurogenic differentiation of adipose-derived MSCs appear under influence of specific inductors of differentiation - retinoic acid or azacitidine and other neurotrophic factors. In particular, the retinoic acid stimulates production of functionally immature neurons with an undeveloped synaptic network in the absence of their membranes excitability. The described method of neurospheres induction from rat adipose-derived MSCs followed by differentiation into neuronal-, glial- and Schwann-like cells.

Our study demonstrated that using the methods of cell isolation from adipose tissue of experimental animals and humans with protocols of long-term culture allow obtain the fraction of MSCs-like cells. As showed by the cytological analysis of culture, adipose-derived cell acquire phenotypic signs of the MSCs in the dynamic. They acquire the fibroblast-like phenotype, manifest adhesive properties and the capacity for proliferation and plasticity. Addition of retinoic acid or 5-azacitidine to the culture medium stimulates the appearance of cytologic signs of the directed neuroglial differentiation and expression of typical for neurons and astrocytes phenotype with formation of neural tissue-like network. This has been confirmed by the immunohistochemical detection of the specific cell markers.

\section{To be still young: role of heme oxygenase-1 in hematopoietic stem cells}

\author{
Szade K., Zukowska M., Nowak W.N., Szade A., Bukowska-Strakova K., Kachamakova-Trojanowska N., Dulak J., Цozkowicz A. \\ Jagiellonian University, Krakow, Poland \\ e-mail: alicja.jozkowicz@uj.edu.pl
}

Heme oxygenase-1 (HO-1) regulates the response of hematopoietic stem cells (HSC) to acute stress. Our aim was to investigate whether H0-1 influences the HSC function in a steady state conditions.

We found that H0-1 in HSC (defined as LincKit+Sca1 ${ }^{+}$CD48-CD150+CD34- cells) is localized mainly in the nucleus where it forms foci, especially in aged animals. HSC from young $\mathrm{HO}-1$ deficient $\left(\mathrm{HO}-1^{-/}\right)$mice lose quiescence, extensively proliferate and contain a higher fraction of $\mathrm{YH} 2 \mathrm{AX}$-high cells, that may indicate the enhanced DNA damage. Young $\mathrm{HO}-1^{-1-}$ individuals have also more myeloid cells in peripheral blood, and their single sorted HSC show increased myeloid differentiation in vitro. All these features are typical for aged hematopoietic system. Thus, RNA-seq analysis revealed that transcriptome of young $\mathrm{HO}-1^{-1-H S C}$ closely resembles the transcriptome of old $\mathrm{HO}-1+/+\mathrm{HSC}$, with deregulated cell cycle checkpoints, symptoms of replication stress, enhanced translesion DNA synthesis, and activated DNA damage response accompanied by DNA repair based on Fanconi anemia and homologous recombination pathways. Finally, when we transplanted purified $\mathrm{HSC}$ from $\mathrm{HO}-1^{-1-}$ donors to $\mathrm{HO}-1^{-1-}$ recipients they showed clearly impaired repopulation capacity in all tested lineages.

The expression of H0-1 in HSC was relatively low. Much higher level we found in the HSC niche, especially in reticular cells and CD31+Sca1+ endothelial cells localized to the bone metaphysis, suggesting that H0-1 may be even more important in the niche than in HSC themselves. Indeed the GO enrichment analysis showed that lack of $\mathrm{HO}-1$ affects genes involved in integrin-mediated cell adhesion and genes regulating

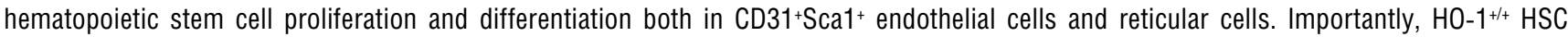
transplanted to $\mathrm{HO}-1^{-/}$animals gave worse reconstitution of peripheral blood after 32 weeks and were unable to reconstitute any $\mathrm{HO}-1^{+/+}$ secondary recipient. On the other hand, transplantation of $\mathrm{HO}-1^{-/-} \mathrm{HSC}$ to the $\mathrm{HO}-1^{+/+}$niche preserved their repopulation capacity in $\mathrm{HO}-1^{+/+}$ secondary recipient. of HSC.

Concluding, $\mathrm{HO}-1$ prevents $\mathrm{HSC}$ from premature aging and $\mathrm{HO}-1$ expression in the niche is necessary for proper reconstitution capacities 


\title{
Effects of cell population enriched with serotonin-producing progenitors on the regeneration of nuclei raphe during in vitro injury
}

\author{
Vasyl'ieva I. H., Oleksenko N. P., Chopyk N. G., Tsiubko O. I., Halanta O. S., Snitsar N. D., Shuba I. M. \\ A. P. Romodanov State Institute of Neurosurgery NAMS of Ukraine, Kyiv, Ukraine \\ e-mail: N.Oleksenko@gmail.com
}

The prognosis of the brain structures traumatic injury outcomes, especially in the neuromediator systems, is complicated in view of their interaction. Using in vitro modelling of mechanic trauma of nuclei raphe we studied the reaction of serotoninergic system on traumatic injury and the regenerative trophic effects of fetal cells.

MATERIAL AND METHODS. In experiment we used the long-term (7 weeks) nuclei raphe tissue culture of the newborn rats isolated with maximal maintenance of intercellular junctions in the sagittal section. Trauma was performed by mechanical section of the neuroglial fibers.

RESULTS. This model caused degenerative changes in the tissue like destruction with large number of detritus in the cutting area and in the distant zones. Histochemical staining to serotonin and expression of the main marker genes of serotonin production (Dlx, Pet1, Nkx2.2, Tph1 and Sert) was negative. During further cultivation we observed no renewal of neuroglial fibers, but only progress of the morphological and functional degeneration was detected.

In the parallel group after neurotrauma model we added to the culture serotonin-activated suspension of the fetal nuclei raphe cells. This cell population displayed a high level of Nkx2.2 gene expression, evidencing for enrichment by serotonin-producing progenitors. Our investigations showed the renewal of destructed neuroglial fibers and their growth across the cutting zone. This process was accompanied with the appearance of serotonin granules in the new-formed processes. Its overall amount in the conditioned medium, according to ELISA data, increased 1.4-fold. The gene analysis demonstrated the expression of serotonin-related genes, including Tph and Sert.

CONCLUSION. Thus, by the in vitro modelling of serotoninergic conduction tracts neurotrauma the addition of serotonin-producing population of the fetal cells stimulated the morphologic and functional regeneration involving of the respective regulatory genes.

\section{In vitro study of cryopreserved multipotent mesenchymal stromal cells from bone marrow, adipose tissue and tendons}

\author{
Volkova N. O., Yukhta M. S., Goltsev A. M. \\ Institute for Problems of Cryobiology and Cryomedicine NAS of Ukraine, Kharkiv, Ukraine \\ e-mail: volkovanatali2006@yandex.ru
}

The demand on new methods for regeneration of damaged tissues and organs is rising in modern biotechnology. Among the perspective methods in clinical practice there is a use of the cryopreserved specialized and stem cells obtained from various sources. Modern technologies of cell culture and cryopreservation allow making cell reserve for long-term storage at low temperatures, transportation and thawing directly before therapeutic application.

Aim. It was a carrying out of comparative analysis of the multipotent mesenchymal stromal cells (MMSCs) from the bone marrow, adipose tissue and tendons in vitro.

Material and methods. The MMSCs' cultures obtained from the bone marrow, adipose tissue and tendons of the rats were cryopreserved in medium contained $10 \%$ DMSO and $20 \%$ FBS with cooling rate of $1{ }^{\circ} \mathrm{C}$ per minute till $-80^{\circ} \mathrm{C}$ and storaged in liquid nitrogen. Thawing was done on water bath at $40^{\circ} \mathrm{C}$ until the appearance of liquid phase. Proliferative characteristics were explored using the MTT-test on day 1,3 and 10 . The MMSCs' ability for migration was determined by the fill rate of defect in monolayer $\left(0.2 \mathrm{~cm}^{2}\right)$ within 3 days. Staining for collagen type I was performed using monoclonal antibodies to type I collagen (1:2000, Sigma-Aldrich, USA) and CF ${ }^{\mathrm{TM}} 488 \mathrm{~A}$ (Sigma-Aldrich, USA). Data analysis was performed by means of the one-factor analysis and Student's t-test using MS Excel and Statistica 8 software.

Results. The cryopreserved MMSCs from bone marrow, adipose tissue and tendons keep their abilities for adhesion and proliferation. The cryopreserved MMSCs from bone marrow were characterized by slow proliferation in culture $(67.2 \pm 4.5 \%$ of the monolayer on the day 10$)$, low migration ability and high level of type I collagen synthesis $(89.6 \pm 2.7 \%$ of the positive cells). The cryopreserved MMSCs' cultures from the adipose and tendon tissues versus bone marrow MMSCs had high proliferative activity $(90.5 \pm 3.1 \%$ and $96.2 \pm 2.7 \%$ of the monolayer on day 10, respectively). Synthesis of type I collagen in MMSCs from the adipose and tendon tissues was lower than in bone marrow MMSCs making $52.6 \pm 2.1 \%$ and $63.4 \pm 2.7 \%$ of positive cells, respectively. Low proliferative activity and high level of the type I collagen expression are characteristic of the cryopreserved MMSCs from the bone marrow. The MMSCs from the adipose tissue and tendons in the analogous conditions of cryopreservation have a higher ability for proliferation and a lower synthetic activity in the comparison with the MMSCs from bone marrow.

Conclusion. Although the findings of our study have no direct clinical implication, in our opinion, the MMSCs obtained from bone marrow, adipose tissue and tendons have a great promise for their application in regenerative medicine. 